\title{
Joint and unique multiblock analysis of biological data - multiomics malaria study $\dagger$
}

\author{
Izabella Surowiec, ab Tomas Skotare, iD a Rickard Sjögren, (D) ab \\ Sandra Gouveia-Figueira, a Judy Orikiiriza, ${ }^{\text {cde }}$ Sven Bergström, \\ Johan Normark ${ }^{f}$ and Johan Trygg (iD *ab
}

Received 18th December 2018, Accepted 8th February 2019

DOI: $10.1039 / c 8 f d 00243 f$

Modern profiling technologies enable us to obtain large amounts of data which can be used later for a comprehensive understanding of the studied system. Proper evaluation of such data is challenging, and cannot be carried out by bare analysis of separate data sets. Integrated approaches are necessary, because only data integration allows us to find correlation trends common for all studied data sets and reveal hidden structures not known a priori. This improves the understanding and interpretation of complex systems. Joint and Unique MultiBlock Analysis (JUMBA) is an analysis method based on the OnPLS-algorithm that decomposes a set of matrices into joint parts containing variations shared with other connected matrices and variations that are unique for each single matrix. Mapping unique variations is important from a data integration perspective, since it certainly cannot be expected that all variation co-varies. In this work we used JUMBA for the integrated analysis of lipidomic, metabolomic and oxylipins data sets obtained from profiling of plasma samples from children infected with $P$. falciparum malaria. P. falciparum is one of the primary contributors to childhood mortality and obstetric complications in the developing world, which makes the development of new diagnostic and prognostic tools, as well as a better understanding of the disease, of utmost importance. In the presented work, JUMBA made it possible to detect already known trends related to the disease progression, but also to discover new structures in the data connected to food intake and personal differences in metabolism. By separating the variation in each data set into joint and unique, JUMBA

\footnotetext{
${ }^{a}$ Computational Life Science Cluster (CLiC), Department of Chemistry, Umeå University, Linnaeus väg 10, 901 87 Umeå, Sweden. E-mail: johan.trygg@umu.se; Tel: +46730647137

${ }^{b}$ Sartorius Stedim Data Analytics, Tvistevägen 48, 90736 Umeå, Sweden

'Infectious Diseases Institute, College of Health Sciences, Makerere University, P.O. Box 22418, Kampala, Uganda

${ }^{d}$ Department of Immunology, Institute of Molecular Medicine, Trinity College Dublin, St. James's Hospital, Dublin 8, Ireland

${ }^{e}$ Rwanda Military Hospital, P.O. Box: 3377, Kigali, Rwanda

${ }^{f}$ Department of Molecular Biology, Umeå University, 90187 Umeå, Sweden

$\dagger$ Electronic supplementary information (ESI) available. See DOI: 10.1039/c8fd00243f
} 
reduced the complexity of the analysis and facilitated the detection of samples and variables corresponding to specific structures across multiple data sets, and by doing this enabled fast interpretation of the studied system. All of this makes JUMBA a perfect choice for multiblock analysis of systems biology data.

\section{Introduction}

Malaria remains a major global health and economic burden in spite of recent intense preventive measures, with the infection caused by the parasite being one of the primary contributors to childhood mortality and obstetric complications in the developing world. ${ }^{1}$ The biochemical mechanisms behind malaria pathogenesis and the impact of the parasite on the host response are still largely unknown. To decipher the underlying mechanisms of malaria, a holistic systems biology approach is crucial. Systems biology aims at a higher level of understanding of organisms by studying them as integrated systems of genetic, protein, metabolic, pathway and cellular events. Analysis of complex biological processes within a systems biology approach is now possible to achieve thanks to the extensive development of a range of 'omics' technologies (genomics, transcriptomics, proteomics, metabolomics, and beyond). ${ }^{2}$ All 'omics' technologies provide massive amounts of data, making analysis highly challenging and requiring powerful computational methods.,4 ${ }^{3,4}$ overcoming these challenges and using systems biology to understand the host-parasite relationship in malaria may lead to new ways of treating the malaria infection.

Systems biology requires integrated analysis of multiple data sets. Although analysis of a single data set is a solved problem, integrated analysis of several different types of data sets, also called blocks, is challenging. At the same time, integration reveals previously unknown hidden structures across multiple data sets and detects samples and variables corresponding to them. Combining information from many data sets can also improve interpretation of the trends observed in the studied system. There exists a wide range of methods for integrated analysis, including methods based on network analysis, ${ }^{5,6}$ Bayesian factor analysis $^{7}$ and multivariate linear projections. ${ }^{8-10}$ In this article we used Joint and Unique MultiBlock Analysis (JUMBA), ${ }^{11,12}$ which is a multivariate linear projection method. Such methods handle noisy, multicollinear data with many more variables than observations (samples), which is typical for biochemical and biological applications.

JUMBA is based on the OnPLS-algorithm ${ }^{\mathbf{8}, 13}$ and is used to perform unsupervised integration of multiple data sets, so called multiblock analysis. Multivariate linear projection methods such as JUMBA have long been used for both unsupervised analysis, for instance Principal Components Analysis (PCA), as well as supervised analysis, for instance Orthogonal Projects to Latent Structures (OPLS). ${ }^{14}$ Although methods such as PCA can be used to analyse multiple blocks by combining the matrices into one ${ }^{15,16}$ they do not distinguish between variation that is joint between blocks and unique variation, which makes interpretation difficult. To perform unsupervised integration of two blocks, O-PLS was modified into O2-PLS, ${ }^{17,18}$ which was later generalized into OnPLS. ${ }^{8,13}$ OnPLS is an algorithm that separates data matrices into variation joint between all or only some blocks as well as variation unique to each block. JUMBA is an OnPLS workflow 
that structures multiblock analysis via pre-processing, modelling, validation, visualization and interpretation of the data. ${ }^{\mathbf{1 1 , 1 2}}$

There are several examples of the application of multiblock analysis based on multivariate linear projection methods in systems biology. O2-PLS has been used for combined modelling of transcript, protein and metabolite data in plant species, ${ }^{19,20}$ for multiblock analysis of fatty acid and lipid profiles in a mice model of familial dysbetalipoproteinemia ${ }^{21}$ and for integration of NMR and DIGE data from prostate cancer xenograft mice. ${ }^{22}$ OnPLS has been used for the integration of transcriptomic, proteomic and metabolomic data for the global investigation of stress response ${ }^{23}$ and secondary cell wall synthesis ${ }^{24}$ in Populus plants, as well as for transcriptomics, metabolomics, sphingolipids, oxylipins, and fatty acids interrogation of biological interactions in asthma. ${ }^{25}$ In all cases, integrated analysis increased the interpretability of the models and enabled important biological conclusions, which would not be possible to achieve with the application of other methods.

We have recently applied metabolomics profiling on plasma from children infected with malaria and showed that a metabolite signature could be used for decision support in disease staging and prognostication, with fatty acids being potential biomarker molecules. ${ }^{26}$ The study was later expanded to the analysis of another set of samples and application of an additional two platforms: LCMS lipid $^{27}$ and oxylipin profiling. ${ }^{28}$ In these studies, we showed that the malaria infection altered the lipid and oxylipin patterns and that these changes could be connected to energy turnover and immune regulation. In the present study we wanted to see if we can confirm the findings from the analysis of separate data sets by application of a faster, integrative approach and to investigate if such an approach can provide more information about unknown trends/structures in the data that can be used for enhanced interpretation of the studied data sets. With this in mind, we have used JUMBA for multiblock analysis on lipidomic, metabolomic and oxylipins data sets obtained from profiling of plasma samples from children infected with $P$. falciparum malaria.

\section{Experimental methods}

\section{Samples}

Twenty plasma samples from each group of diagnostic categories: healthy controls, mild and severe malaria patients, were chosen from the 690 available, based on the clinical information using a full factorial design, as described before. ${ }^{26}$ The research was carried out according to The Code of Ethics of the World Medical Association (Declaration of Helsinki). Ethical clearance was obtained from the Rwanda National Ethics Committee RNEC (no. 279/RNEC/2010) and the Regional Ethical Committee in Umeå (no. 09-064). Written informed consent was provided by the parent or legal guardian of each participant.

\section{Data sets}

Samples were extracted and analyzed with the GCMS metabolomics profiling method, ${ }^{29}$ with LCMS metabolomics and lipidomics profiling method $\mathrm{s}^{27}$ and with oxylipin targeted LCMS profiling. ${ }^{28}$ For easy interpretation, and due to the large overlap between detected compounds and the fact that they were extracted in the 
same sample preparation step, metabolomics GCMS and LCMS data (from both ionization modes) were combined into one metabolomic data set. For compounds that were common between platforms or between LCMS ionization modes, the ones with lower relative standard deviations in the pooled samples were kept in the table. The applied analytical procedures are given in ESI, File $1 . \dagger$

\section{Data normalization, transformation and scaling}

For the metabolomic data set, the compound peak areas were normalized using areas of internal standards, according to the following procedure: the PCA (with Unit Variance (UV) scaling without subtraction of the mean) on the peak areas of internal standards was calculated and the first component score value for each sample was used to normalize the resolved data by dividing the peak areas of each sample with the corresponding score value. ${ }^{30}$ The oxylipins data set was logtransformed and all three data sets were mean centered and scaled to unit variance before JUMBA analysis.

\section{Joint and unique multiblock analysis - JUMBA}

JUMBA is a workflow for analyzing the underlying structures shared between multiple blocks of data measured on the same set of samples. JUMBA is based on the OnPLS-algorithm that decomposes the variation of data sets into joint and unique variation as well as residuals. Joint variation may be in common in all analyzed blocks, globally joint, or joint between some but not all, locally joint. A schematic overview of a three-block JUMBA model is given in Fig. 1. Even though JUMBA summarizes several blocks at the same time, the components can be

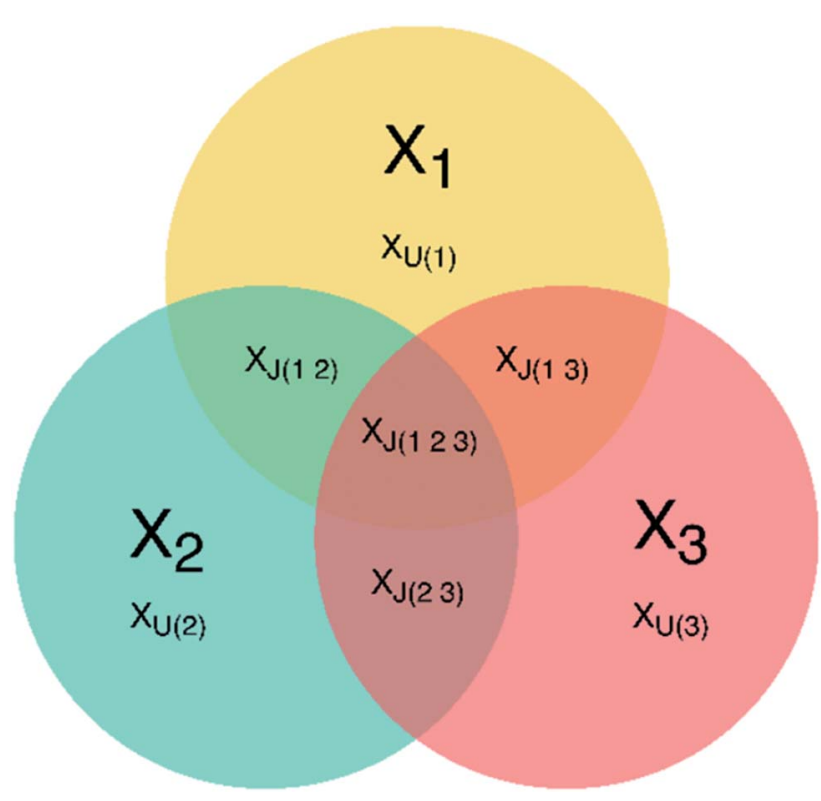

Fig. 1 Overview of a three-block JUMBA model showing possible partition of the variation within three data sets: $X_{J}$ - joint variation, $X_{U}$ - unique variation, 1, 2, 3 - number of data block. 
investigated using scores and loadings in the same way as single-block methods, such as PLS or PCA. The scores show patterns among samples, and can be used to identify trends and outliers, while loadings show correlations between studied variables and their influence on the distribution of samples.

OnPLS, and therefore JUMBA, uses so called block-scores, meaning that each observation is assigned scores for each block instead of a single score per component across all blocks, which is the case for other multiblock methods, like, for example, JIVE and DISCO. ${ }^{31,32}$ The OnPLS-algorithm finds such block-scores that maximize the covariance across all blocks. Separate scores for each block make it possible to investigate how well samples correspond across blocks and may be used to identify observations that correspond poorly between blocks. A single score for each observation gives the impression of artificially strong correspondence across blocks, which limits interpretation.

In this study we used JUMBA ${ }^{11}$ to do multiblock analysis; we used the correlation matrix plot, multiblock scatter plot, explained variance plot and external responses correlation plot to evaluate and visualize the model. To visualize loadings, we used the correlation loadings, $\mathrm{p}$ (corr), since correlation loadings are scaled to correlations in the range -1 to 1 , which simplifies interpretation.

The implementation of the algorithm was done using Mathworks MATLAB. The Pearson correlations were calculated using an in-house script written using the Anaconda Python distribution v. 3.5 (https://continuum.io) and plotted using the Matplotlib library (http://matplotlib.org/). Pathway enrichment analysis was performed on the $\mathrm{p}$ (corr) values above $0.2 \mathrm{using}$ MetaboAnalyst 3.0. ${ }^{33}$

\section{Results and discussion}

\section{Data analysis and pre-treatment}

The fundamental requirement for the application of JUMBA is that there are two or more data sets where the observations can be matched in a $1: 1$ fashion. Similar to other multivariate models, JUMBA is sample efficient and can handle noisy and collinear variables, meaning that it is well suited for, but not limited to, omics data. In data with very strong global variation patterns, as few as twelve observations can be successfully modelled, but inclusion of more observations is recommended. The number of samples included in this study was sufficient to produce good results.

Evaluation of the raw data before JUMBA is important, since disturbances within the data (such as strong outliers) will have a profound impact on the resulting model. In addition to platform specific quality control, single-block analysis of each block prior to multiblock analysis is recommended. The purpose of this is to determine suitable pre-processing for each block, detect and handle outlier observations and variables, and to preliminarily identify trends in each block. During the preliminary data check, two severe malaria samples turned out to have significantly different chromatograms in the GCMS analysis and one mild sample was lost during LCMS analysis, meaning that we removed these three samples from the final data sets. The final data sets consisted, therefore, of 57 samples, each characterized by 100 metabolites, 144 lipids and 37 oxylipins.

Skewness analysis revealed that many variables were not normally distributed, which was especially profound for the oxylipins data set, where no variable passed 
the normality test. Due to the skewed variable distributions, we chose to use logtransformation for the oxylipins data set before JUMBA. Data transformation influences the interpretation of the results and should therefore be used with caution. ${ }^{34}$ In our case, log transformation helped to obtain a less skewed distribution of samples for the oxylipins data set and hence eliminated the need to remove a number of samples due to their what seemed to be deviating behaviour.

\section{JUMBA}

General model structure. Using JUMBA, we found three globally joint components explaining $48.0 \%$ of variation in the lipids data set $(26.6 \%, 12.9 \%$ and $8.5 \%$ of variation distributed between the first, second and third globally joint components, respectively), $38.9 \%$ variation in the oxylipins data set $(16.0 \%$, $17.7 \%$ and $5.2 \%$ ) and $32.7 \%$ in the metabolic data set (11.4\%, $11.8 \%$ and $9.6 \%)$. This means that there was a large overlap between the studied data sets. We found two locally joint components between the metabolomic and oxylipins data sets, describing $4.8 \%, 5.4 \%$ and $7.1 \%, 7.2 \%$ of variation, respectively. We also found four unique components in the lipids data set, $(11.7 \%, 9.2 \%, 8.4 \%$ and $4.2 \%$ of variation explained). In the metabolomic data set we found five unique components $(7.5 \%, 5.2 \%, 4.5 \%, 4.2 \%$ and $3.9 \%$ of variation explained). For the oxylipins data set we also found five unique components $(11.3 \%, 6.6 \%, 3.9 \%, 3.5 \%$ and $3.4 \%$ of variation explained). The remaining variation corresponded to residuals and was equal to $18.6 \%, 18.2 \%$ and $31.8 \%$ of variation for the lipidomic, oxylipins and metabolomic data sets, respectively.

Scores and loading values for all model components are given in ESI, File $2 . \dagger$

Model evaluation. To evaluate the validity of JUMBA, we inspected the correlation matrix plot (Fig. 2), which is a good tool for the detection of problems in the model. ${ }^{11}$ Correlation between different model components may indicate that variation is assigned to joint and unique components in an inappropriate manner. For instance, if a unique component and a joint component correlate significantly, it may indicate that the unique component should actually be part of the joint component. In our case, there were no significant correlations between the different model components, but at the same time there were some correlations between different components (the plot was not too 'clean'), showing that the model was not over fitted, and hence was valid.

Analysis of the globally joint variation. An overview of the sample distribution, as described by the first two globally joint components, is presented on a multiblock scatter plot in Fig. 3. The first globally joint component describes the separation between the controls and the samples from the malaria patients. The second and third globally joint components (not shown) explain within-class variation.

The loadings corresponding to the first globally joint component (Fig. 4) revealed that the main observed compounds that co-varied and were up-regulated in infected individuals were phosphatidylcholines, sphingomyelins and the majority of triacylglycerides (lipids data set), $67 \%$ of all detected metabolites in the metabolites data set, as well as the majority of compounds from CYP, four compounds from 5-LOX and one compound (13-oxo-ODE) from the 12/15-LOX synthesis pathway (oxylipins). The main compounds that co-varied and were down-regulated in infected individuals were lysophosphatidylcholines (lipids) 


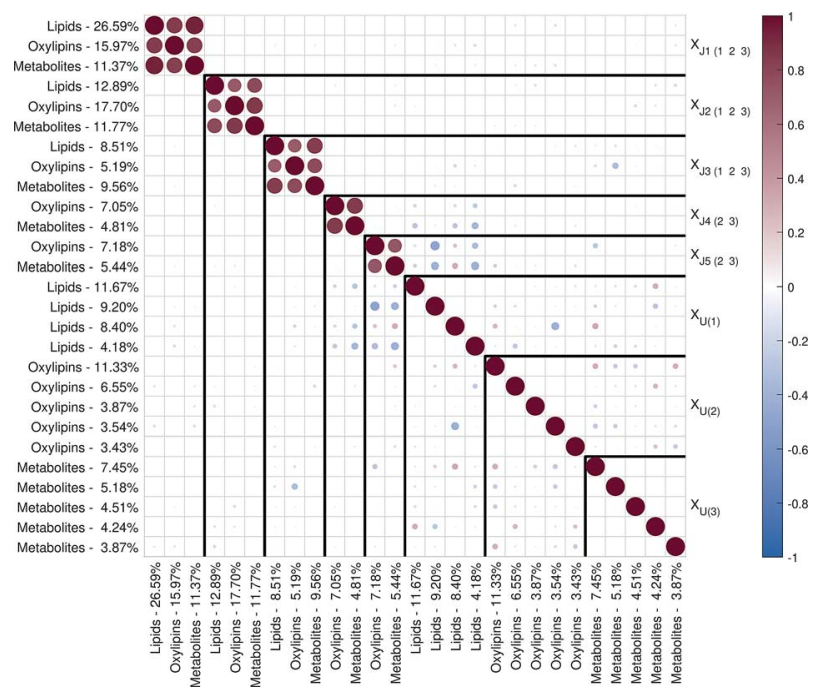

Fig. 2 Correlation matrix plot for the malaria study; the color and area of the circles correspond to the sign and strength of the correlation, with increasing circle size and color intensity indicating increasing correlation (positive (red) or negative (blue)). The thick vertical and horizontal lines are a visual aid to see the distinction between model components and unique component blocks; ${ }^{11} \mathrm{~J}$ - joint component, $U$ - unique component.

and oxylipins from the COX and 12/15-LOX synthesis pathways (oxylipins). These results are consistent with the ones obtained from the OPLS-DA analysis of differences between patients and controls performed for separate data sets. ${ }^{27,28}$ This confirms that JUMBA can be used for the fast, successful integration and extraction of relevant information from the multiblock data.

While finding correlations between different data sets corresponding to the known sample groups by studying data sets separately and then combining the results is possible to some extent, analysis of any trends in common between blocks that are not known a priori can only be carried out using an integrated approach. In this study we have focused on the analysis of the second and third globally joint, as well as locally joint vectors, to elucidate such trends.

None of the trends observed in the second and third global components could be contributing to the previously known information about the samples. The loadings for the second globally joined vector, Fig. 5, show that the main trend observed with positive scores of the second globally joint component was downregulation of the majority of phospholipids and triacylglycerides with lower carbon contents and higher degrees of saturation (lipids), down-regulation of amino acids with higher levels of fatty acids (metabolites) and higher levels of practically all oxylipins. The observed metabolic and oxylipin profiles correspond well to the ones observed in human plasma after the intake of a defined meal. ${ }^{35,36}$ Also, postprandial dyslipidemia is a well described phenomenon. ${ }^{37}$ Since samples included in this study were fed ad libitum, it is possible that some of them were taken shortly after a meal, and that the response to food intake represents part of the within-group variation described by the second globally joint component. 


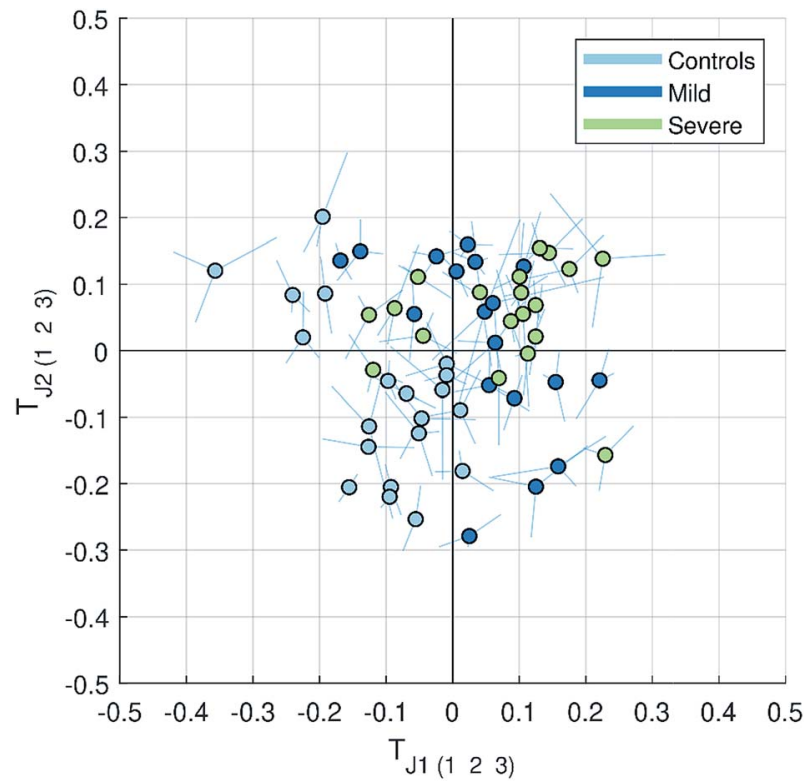

Fig. 3 Multiblock scatter plot made for the JUMBA model showing how the observations varied across several blocks for the globally joint components $T_{\mathrm{J}_{1}}$ and $T_{\mathrm{J}_{2}}$. The samples are colored according to the class they belong to (controls, mild and severe malaria). The multiblock scatter plot is created by normalizing the score vectors to equal length, averaging the score values across blocks sample-wise and plotting them on a two dimensional plot as a mean score of each observation (sample), with lines drawn to each original block score value. ${ }^{11} \mathrm{~A}$ point with longer lines in comparison to others will correspond to the observation with a large variation in the score values.

Connection of the second joint component to the food intake can also be supported by the size of the variation in the data connected to this component a similar size for the oxylipins and metabolic data sets and approximately two times lower for the lipidomic data set as compared to the variation connected to the first globally joined vector. It is reasonable to assume that the intake of a meal will have a large impact on the metabolic and lipid composition of plasma. As such, analysis of the second globally joint vector provided additional information about the trends common for the studied data sets, which would be difficult, if not impossible, to gain by the analysis of separate blocks.

Analysis of the locally joint variation. Whereas globally joint components represent trends in the data common for all data sets, the locally joint ones correspond to the variation that is shared between a few but not all data sets. This distinction between different ways of sharing of variation across data sets is difficult to detect with other statistical methods. In our case, JUMBA found a share of the total variation that was joined between the metabolic and oxylipins data sets, split into two components. Since the samples for these data sets were prepared and analysed using different protocols, joint variation is probably connected to the intrinsic properties of biological, rather than experimental, origin. We could not find any clear trends linking to known sample descriptors by inspecting the score plot of joint score vectors. Pathway analysis of the 

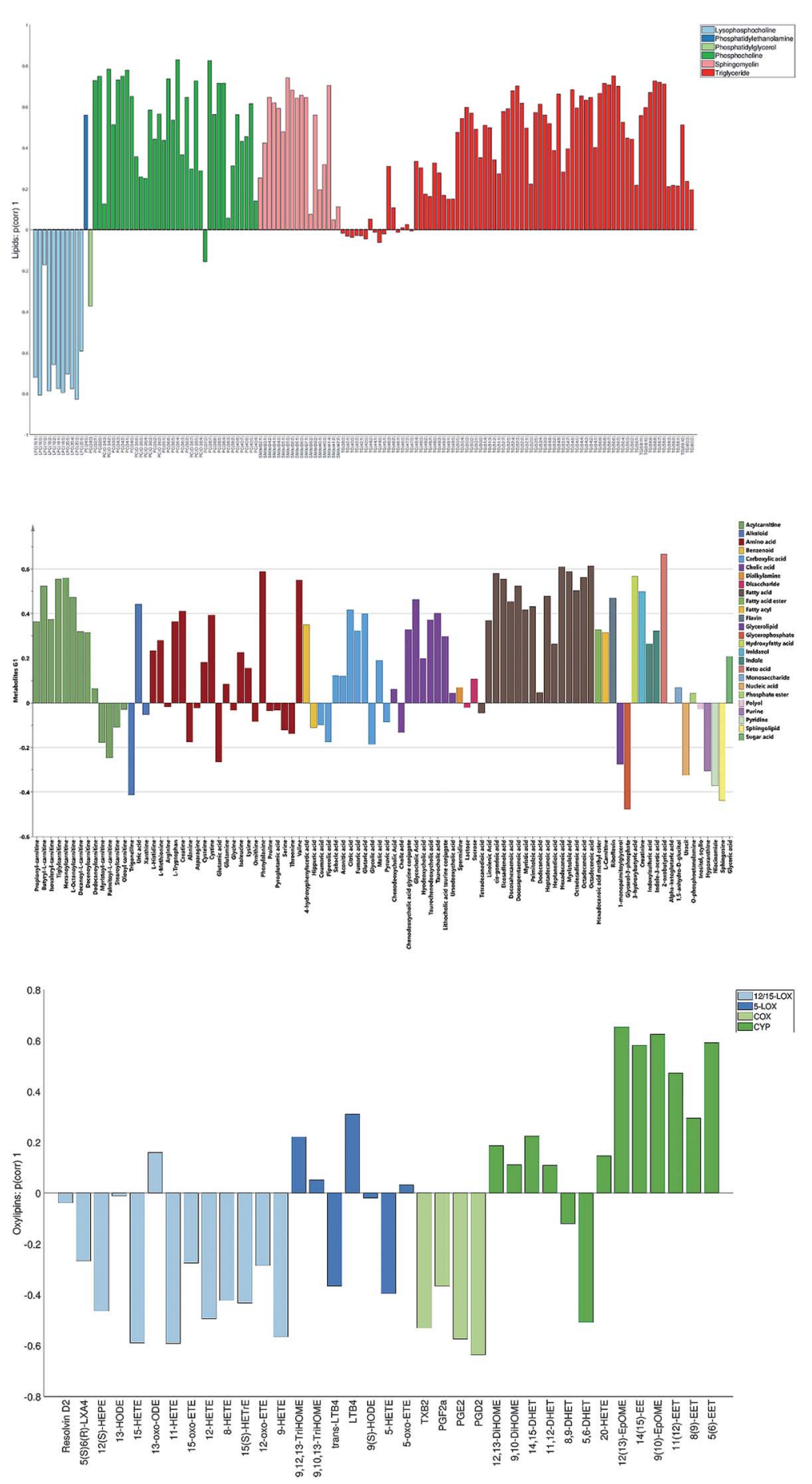

Fig. 4 First globally joint loading vectors (p(corr)) for the lipids (A), metabolites (B) and oxylipins (C) data sets colored according to the chemical classes (metabolites and lipids) and biochemical synthesis pathways (oxylipins). 

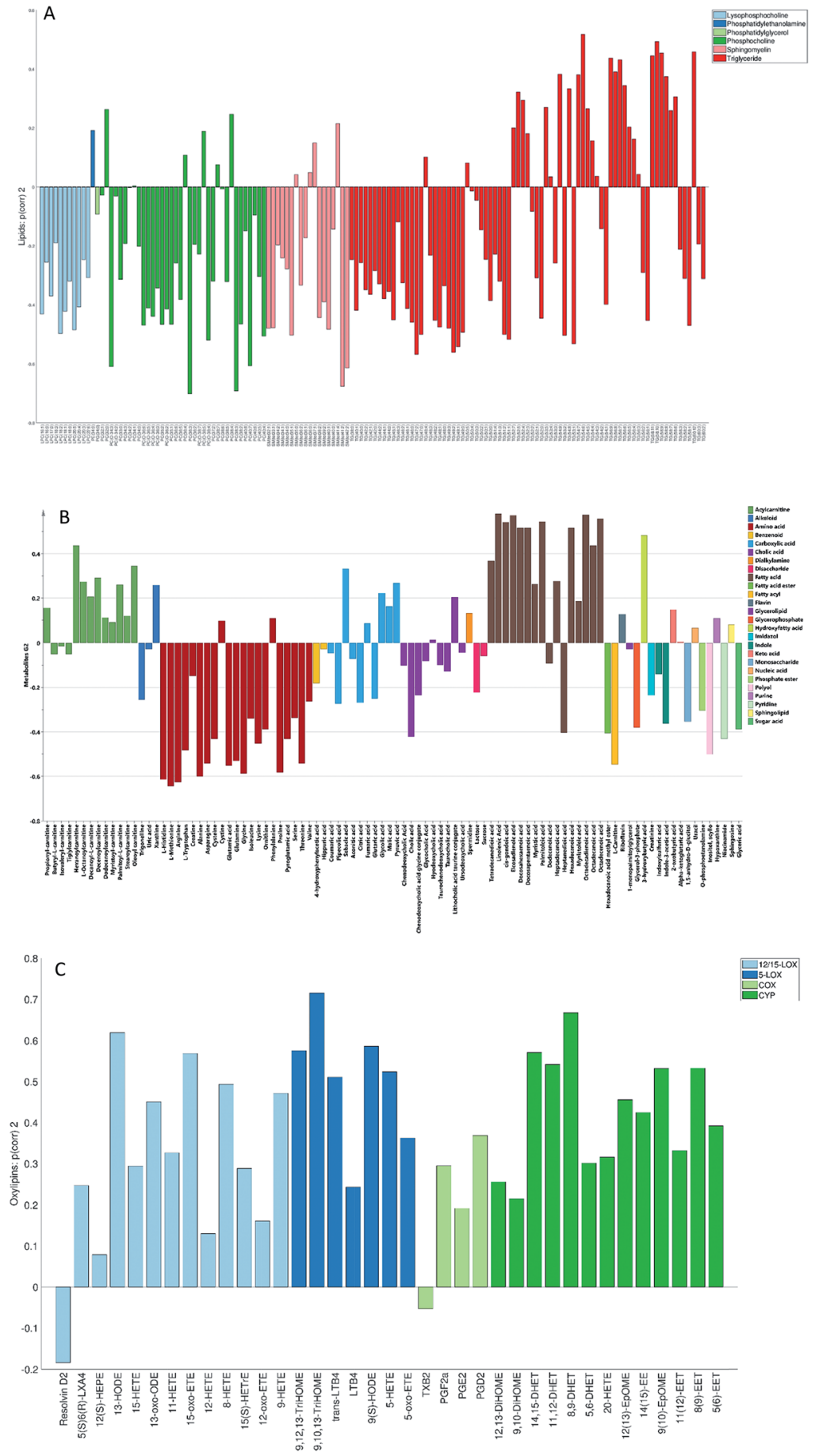

Fig. 5 Second globally joint loading vectors (p(corr)) for the lipids (A), metabolites (B) and oxylipins (C) data sets coloured according to the chemical classes (metabolites and lipids) and biochemical pathways (oxylipins). 
corresponding loadings (ESI, File 1, Fig. S1 and S2 $\dagger$ ) revealed that linoleic and arachidonic acid metabolism as well as amino acid metabolism and the citric acid cycle were the most affected pathways for the first locally joint component. For the second locally joint component, amino acid and arachidonic acid metabolism, primary bile acid biosynthesis and lysine degradation were most affected. At this point, it is not possible to connect observed metabolic/oxylipin trends to specific physiological processes, but the observed connection to the arachidonic acid metabolism suggests changes in the immunological response not connected to the general metabolic response along the main sick-control axis. This indicates that the locally joint information could be related to a personal response to malaria. Since the results of the pathway analysis are highly related and limited to the capabilities of the platform used for the detection of the compounds (for example, types of compounds that can be detected and ones which will be underrepresented), as well as the cut-off used for the selection of the compounds used as input for the pathway analysis, interpretation of the loadings presented above would need further verification.

Analysis of the unique variation. Unique variation describes the structured variation characteristic for one data set only and may be connected to experimental and/or biological factors. Loading plots corresponding to the selected four unique components are shown in Fig. 6. The first unique loading for the oxylipins data set and the second for the lipids data set showed the majority of compounds having positive and negative $\mathrm{p}$ (corr) values, respectively. Such a trend would rather not be expected from changes in biochemical pathways since this would mean activation of all pathways without any counterbalance effect. As such, trends described by these unique loadings could be most probably connected to experimental bias (for example, extraction errors not fully compensated by normalization of data to internal standards). The first unique loading for the lipids data set was among others characterized by negative $\mathrm{p}$ (corr) values of triacylglycerides with shorter fatty acid chain lengths and higher levels of saturation, which may be connected to personal differences in lipid metabolism or to specific types of diet. For the metabolomic data set, the first unique loading was characterized by all amino acids having positive $\mathrm{p}$ (corr) values and acylcarnitines with fatty acid chain lengths over six carbon atoms and fatty acids having negative $\mathrm{p}$ (corr) values. Long-chain acylcarnitines accumulate in the state of dysregulated fatty acid oxidation, especially during periods of increased energy demand from fat. This means that the observed profile could be connected to fatty acid oxidation defects; a statement needing further verification.

Correlation of the JUMBA components with metadata. The external response correlation plot shows correlations between known sample descriptors and the components of the model, and can be used for interpretation of the model. For this study, correlations of the model components against available personal and clinical parameters of the samples are shown in Fig. 7. The first globally joined component was strongly correlated with the class of the sample 'Controls', as well as with several parameters describing the severity of the sickness, like temperature, breathing rate and pulse rate, as well as symptoms describing the severity of the disease, for example loss of consciousness, convulsions, etc. This confirmed the previously discussed observation that trends described by the first joint component were related to the differences between the infected individuals and the controls. The first unique component for the metabolic data set correlated 

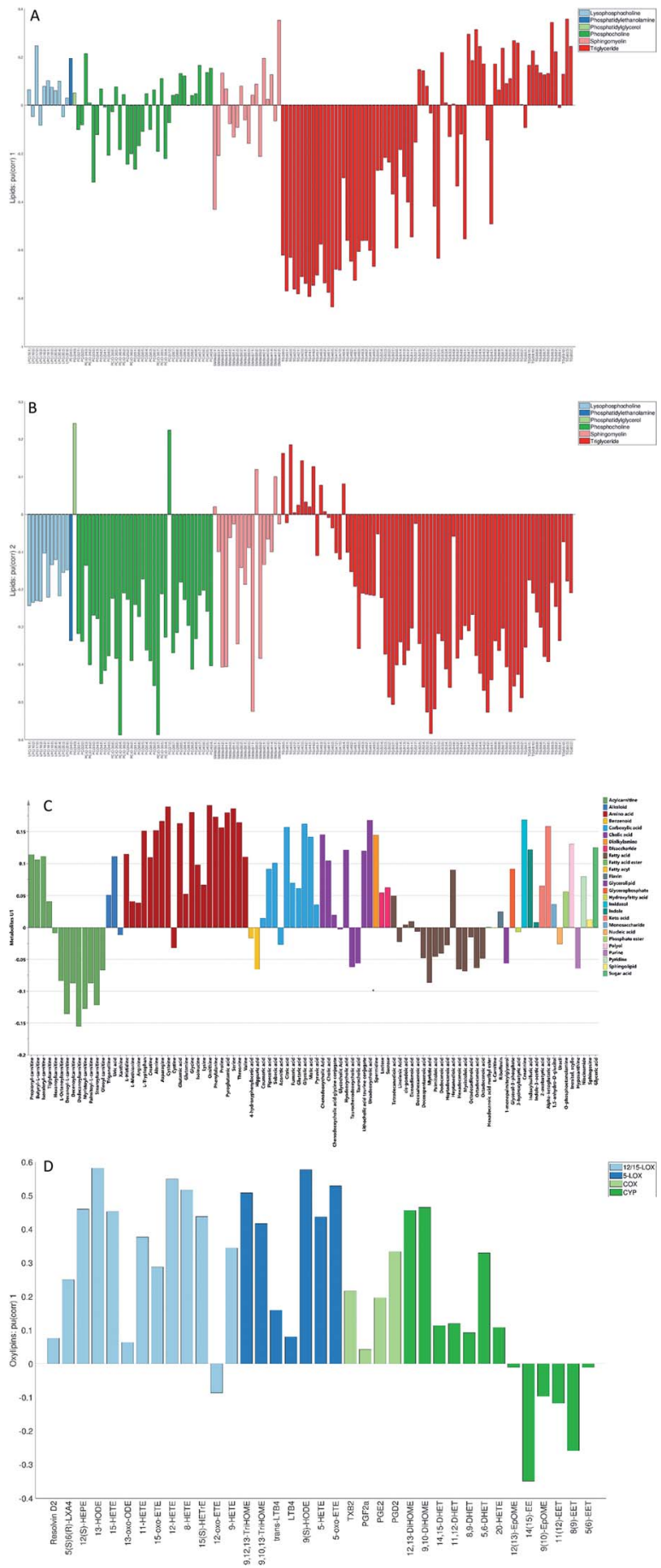

Fig. 6 First unique loading vectors ( $p$ (corr)) for the lipids (A, B), metabolites (C) and oxylipins (D) data sets coloured according to the chemical classes (metabolites and lipids) and biochemical pathways (oxylipins). 


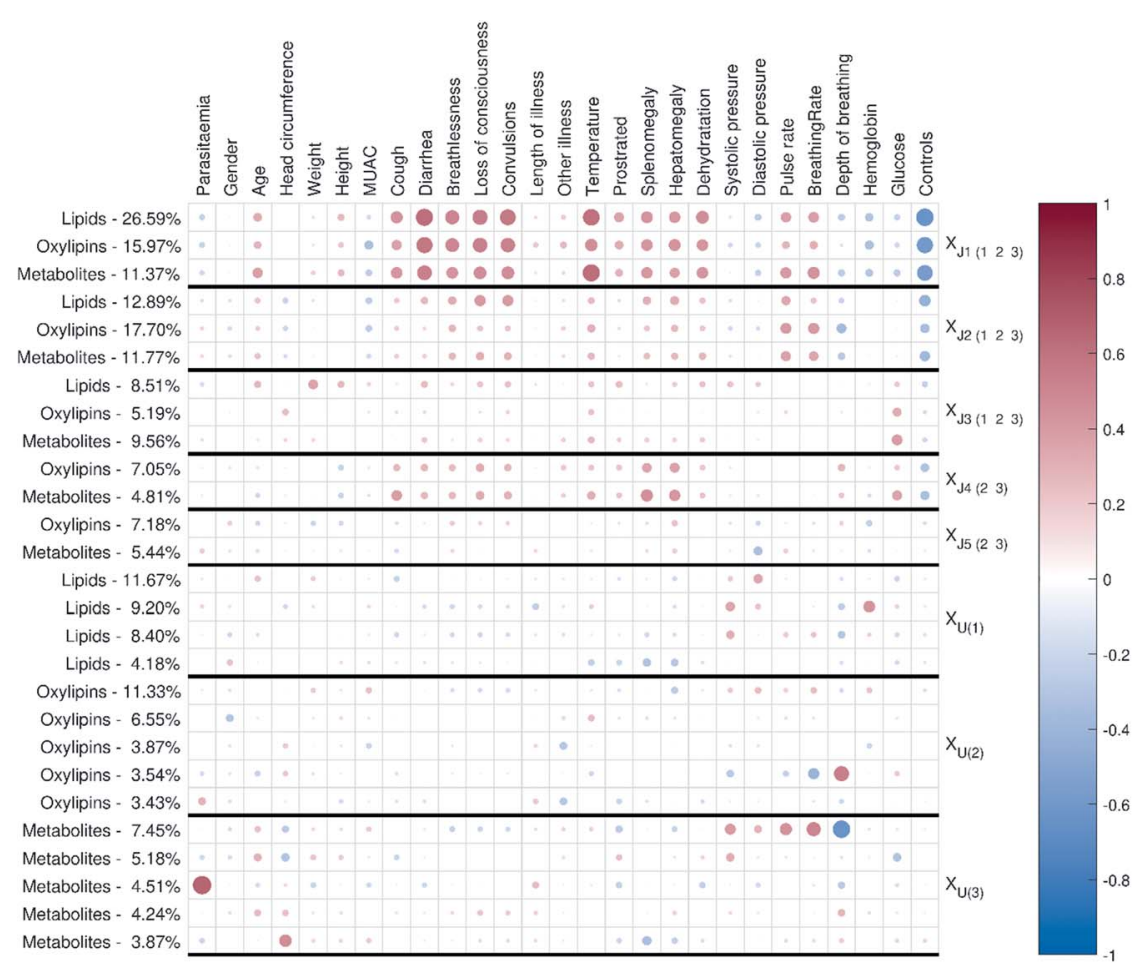

Fig. 7 The external response correlation plot for the malaria study showing correlations between known external response variables (personal and clinical parameters of the samples) and the different components of the model. The colour and size of the circles correspond to the sign and strength of the correlation, with increasing circle size and colour intensity indicating increasing correlation; blue shades are used for negative correlations and red shades for positive correlations.

positively with systolic pressure, pulse rate, breathing rate, hepatomegaly and loss of consciousness. These are parameters related to the severity of the disease, which could be connected to changes in the fatty acid beta-oxidation, as suggested by the analysis of the loading profile of this component.

\section{Conclusions}

In this study we have presented an integrative approach for the combined analysis of multiple data sets in clinical settings. Using JUMBA, we were able to reveal and analyse variation shared between lipidomic, metabolomic and oxylipin profiles of plasma that corresponded to intrinsically linked flows of information. Analysis of the joint loadings confirmed previously known trends in data related to the disease biochemistry and helped to immediately elucidate correlations between the studied data sets relating to these trends. We also revealed previously unknown trends that were most probably related to food intake and personal differences in the immunological response and general metabolism. Detection of those trends would not be possible to achieve by analysing each data set separately. Analysis of the relevant vectors from variations unique to each data block 
provided information specific for each data set that could be interesting both biologically, as well as from the analytical methodology point of view.

We proved that JUMBA can successfully integrate data obtained from different analytical platforms, thanks to its robustness to noise and its compartmentalization of variation into joint and unique parts. Integrated analysis of multiple data sets provides a faster and easier means to visualize and hence interpret the found relationships rather than separate investigation of each data set. Our integrative approach revealed hidden structures in the data not known a priori. It also allowed the detection of samples and variables corresponding to the found structures across multiple data sets, such as food intake, personal response to the disease or platform specific variations. To summarize, JUMBA is an easy-to-use workflow to put multiple data sets together, which offers enhanced visualization and allows comprehensive interpretation of multiblock data. JUMBA is a suitable method for handling complex multi-omics data sets and has high potential to improve the biological understanding of the studied systems. However, as in the case of all multivariate modelling methods, also for the application of JUMBA, common sense is needed. In cases where there are very many and very noisy variables, there is always the risk of spurious correlations, meaning that components can be found just by pure chance. One way to alleviate this risk is to investigate each block separately by evaluating its structure using well-established metrics, like, for example, PCA. Since the OnPLS-algorithm finds linear components, it is not suitable for detecting non-linear relationships. In those cases, we recommend finding feature representations of the data that are able to represent non-linear relationships (for instance, polynomial features).

\section{Conflicts of interest}

There are no conflicts to declare.

\section{Acknowledgements}

We would like to acknowledge the Swedish Metabolomics Centre for support with the chromatographic analysis.

\section{References}

1 P. F. Beales, B. Brabin, E. Dorman, L. Loutain, K. Marsh, M. E. Molyneux, et al., Severe falciparum malaria, Trans. R. Soc. Trop. Med. Hyg., 2000, 94, S1-S90.

2 A. Fukushima, M. Kusano, H. Redestig, M. Arita and K. Saito, Integrated omics approaches in plant systems biology, Curr. Opin. Chem. Biol., 2009, 13(5-6), 532-538.

3 S. E. Richards, M.-E. Dumas, J. M. Fonbille, T. M. D. Ebbels, E. Holmes and J. K. Nicholson, Intra- and inter-omic fusion of metabolic profiling data in a systems biology framework, Chemom. Intell. Lab. Syst., 2010, 104(1), 121-131.

4 A. R. Joyce and B. O. Palsson, The model organism as a system: integrating 'omics' data sets, Nat. Rev. Mol. Cell Biol., 2006, 7(3), 198-210.

5 R. Shen, A. B. Olshen and M. Ladanyi, Integrative clustering of multiple genomic data types using a joint latent variable model with application to 
breast and lung cancer subtype analysis, Bioinformatics, 2009, 25(22), 29062912.

6 B. Wang, A. M. Mezlini, F. Demir, M. Fiume, Z. Tu, M. Brudno, et al., Similarity network fusion for aggregating data types on a genomic scale, Nat. Methods, 2014, $11(3), 333$.

7 R. Argelaguet, B. Velten, D. Arnol, S. Dietrich, T. Zenz, J. C. Marioni, et al., Multi-Omics Factor Analysis-a framework for unsupervised integration of multi-omics data sets, Mol. Syst. Biol., 2018, 14(6), e8124.

8 T. Lofstedt and J. Trygg, OnPLS-a novel multiblock method for the modelling of predictive and orthogonal variation, J. Chemom., 2011, 25(8), 441-455.

9 E. F. Lock, K. A. Hoadley, J. S. Marron and A. B. Nobel, Joint and individual variation explained (JIVE) for integrated analysis of multiple data types, Ann. Appl. Stat., 2013, 7(1), 523-542.

10 M. Schouteden, K. Van Deun, S. Pattyn and I. Van Mechelen, SCA with rotation to distinguish common and distinctive information in linked data, Behav. Res. Methods, 2013, 45(3), 822-833.

11 T. Skotare, R. Sjögren, I. Surowiec, D. Nilsson and J. Trygg, Visualization of descriptive multiblock analysis, J. Chemom., 2018, e3071.

12 T. Skotare, D. Nilsson, S. J. Xiong, P. Geladi and J. Trygg, Joint and Unique Multiblock Analysis for integration and calibration transfer of NIR instruments, Anal. Chem., 2019, 91(5), 3516-3524.

13 T. Lostedt, D. Hoffman and J. Trygg, Global, local and unique decompositions in OnPLS for multiblock data analysis, Anal. Chim. Acta, 2013, 791, 13-24.

$14 \mathrm{~J}$. Trygg and S. Wold, Orthogonal projections to latent structures (O-PLS), J. Chemom., 2002, 16(3), 119-128.

15 S. Wold, N. Kettaneh and K. Tjessem, Hierarchical multiblock PLS and PC models for easier model interpretation and as an alternative to variable selection, J. Chemom., 1996, 10(5-6), 463-482.

16 J. A. Westerhuis, T. Kourti and J. F. Macgregor, Analysis of multiblock and hierarchical PCA and PLS models, J. Chemom., 1998, 32, 301-321.

$17 \mathrm{~J}$. Trygg, O2-PLS for qualitative and quantitative analysis in multivariate calibration, J. Chemom., 2002, 16(6), 283-293.

$18 \mathrm{~J}$. Trygg and S. Wold, O2-PLS, a two-block (X-Y) latent variable regression (LVR) method with an integral OSC filter, J. Chemom., 2003, 17(1), 53-64.

19 M. Bylesjo, R. Nilsson, V. Sirvastava, A. Grönlund, A. I. Johansson, S. Jansson, et al., Integrated Analysis of Transcript, Protein and Metabolite Data To Study Lignin Biosynthesis in Hybrid Aspen, J. Proteome Res., 2009, 8(1), 199-210.

20 M. Bylesjo, D. Eriksson, M. Kusano, T. Moritz and J. Trygg, Data integration in plant biology: the O2PLS method for combined modeling of transcript and metabolite data, Plant J., 2007, 52(6), 1181-1191.

21 G. M. Kirwan, E. Johansson, R. Kleemann, E. R. Verheji, Å. M. Wheelock, S. Goto, et al., Building Multivariate Systems Biology Models, Anal. Chem., 2012, 84(16), 7064-7071.

22 M. Rantalainen, O. Cloarec, O. Beckonert, I. D. Wilson, D. Jackson, R. Tonge, et al., Statistically integrated metabonomic-proteomic studies on a human prostate cancer xenograft model in mice, J. Proteome Res., 2006, 5(10), 26422655.

23 V. Srivastava, O. Obudulu, J. Bygdell, T. Löfstedt, P. Ryden, R. Nilsson, et al., OnPLS integration of transcriptomic, proteomic and metabolomic data 
shows multi-level oxidative stress responses in the cambium of transgenic hipI-superoxide dismutase Populus plants, BMC Genomics, 2013, 14, 893.

24 O. Obudulu, N. Mähler, T. Skotare, J. Bygdell, I. N. Abreu, M. Ahnlund, et al., A multi-omics approach reveals function of secretory carrier-associated membrane proteins in wood formation of Populus trees, BMC Genomics, 2018, 19(1), 11.

25 S. N. Reinke, B. Galindo-Prieto, T. Skotare, D. I. Broadhurst, A. Singhania, D. Horowitz, et al., OnPLS-based multi-block data integration: a multivariate approach to interrogating biological interactions in asthma, Anal. Chem., 2018, 90, 13400-13408.

26 I. Surowiec, J. Orikiiriza, E. Karlsson, M. Nelson, M. Bonde, P. Kyamanwa, et al., Metabolic signature profiling as a diagnostic and prognostic tool in pediatric Plasmodium falciparum malaria, Open Forum Infect. Dis., 2015, 2(2), ofv062.

27 J. Orikiiriza, I. Surowiec, E. Lindquist, M. Bonde, J. Magambo, C. Muhinda, et al., Lipid response patterns in acute phase paediatric Plasmodium falciparum malaria, Metabolomics, 2017, 13(4), 41.

28 I. Surowiec, S. Gouveia-Figueira, J. Orikiiriza, E. Lindquist, M. Bonde, J. Magambo, et al., The oxylipin and endocannabidome responses in acute phase Plasmodium falciparum malaria in children, Malar. J., 2017, 16(1), 358.

29 A. Jiye, J. Trygg, J. Gullberg, A. I. Johansson, P. Jonsson, H. Antti, et al., Extraction and GC/MS analysis of the human blood plasma metabolome, Anal. Chem., 2005, 77(24), 8086-8094.

30 H. Redestig, A. Fukushima, H. Stenlund, T. Moritz, M. Arita, K. Saito, et al., Compensation for systematic cross-contribution improves normalization of mass spectrometry based metabolomics data, Anal. Chem., 2009, 81(19), 7974-7980.

31 E. F. Lock, K. A. Hoadley, J. S. Marron and A. B. Nobel, Joint and individual variation explained (JIVE) for integrated analysis of multiple data types, Ann. Appl. Stat., 2013, 7(1), 523-542.

32 M. Schouteden, K. Van Deun, S. Pattyn and I. Van Mechelen, SCA with rotation to distinguish common and distinctive information in linked data, Behav. Res. Methods, 2013, 45(3), 822-833.

33 J. G. Xia, I. V. Sinlenikov, B. Han and D. S. Wishart, MetaboAnalyst 3.0-making metabolomics more meaningful, Nucleic Acids Res., 2015, 43(W1), W251W257.

34 C. Feng, W. Hongyue, N. Lu and X. M. Tu, Log transformation: application and interpretation in biomedical research, Stat. Med., 2013, 32(2), 230-239.

35 S. Gouveia-Figueira, J. Späth, A. M. Zivkovic and M. L. Nording, Profiling the oxylipin and endocannabinoid metabolome by UPLC-ESI-MS/MS in human plasma to monitor postprandial inflammation, PLoS One, 2015, 10(7), e0132042.

36 M. Karimpour, I. Surowiec, J. Wu, S. Gouveia-Figueira, R. Pinto, J. Trygg, et al., Postprandial metabolomics: A pilot mass spectrometry and NMR study of the human plasma metabolome in response to a challenge meal, Anal. Chim. Acta, 2016, 908, 121-131.

37 V. Higgins and K. Adeli, Postprandial Dyslipidemia: Pathophysiology and Cardiovascular Disease Risk Assessment, eJIFCC, 2017, 28(3), 168-184. 